\title{
Cyclic Derangements
}

\author{
Sami H. Assaf* \\ Department of Mathematics \\ MIT, Cambridge, MA 02139, USA \\ sassaf@math.mit .edu
}

Submitted: Apr 16, 2010; Accepted: Oct 26, 2010; Published: Dec 3, 2010

Mathematics Subject Classification: 05A15; 05A05, 05A30

\begin{abstract}
A classic problem in enumerative combinatorics is to count the number of derangements, that is, permutations with no fixed point. Inspired by a recent generalization to facet derangements of the hypercube by Gordon and McMahon, we generalize this problem to enumerating derangements in the wreath product of any finite cyclic group with the symmetric group. We also give $q$ - and $(q, t)$-analogs for cyclic derangements, generalizing results of Gessel, Brenti and Chow.
\end{abstract}

\section{Derangements}

A derangement is a permutation that leaves no letter fixed. Algebraically, this is an element $\sigma$ of the symmetric group $\mathfrak{S}_{n}$ such that $\sigma(i) \neq i$ for any $i$, or, equivalently, no cycle of $\sigma$ has length 1 . Geometrically, a derangement is an isometry in $\mathbb{R}^{n-1}$ of the regular $(n-1)$-simplex that leaves no facet unmoved. Combinatorially, these are matrices with entries from $\{0,1\}$ such that each row and each column has exactly one nonzero entry and no diagonal entry is equal to 1.

Let $\mathfrak{D}_{n}$ denote the set of derangements in $\mathfrak{S}_{n}$, and let $d_{n}=\left|\mathfrak{D}_{n}\right|$. The problem of counting derangements is the quintessential example of the principle of Inclusion-Exclusion [20]:

$$
d_{n}=n ! \sum_{i=0}^{n} \frac{(-1)^{i}}{i !} .
$$

For example, the first few derangement numbers are 0,1,2, 9, 44, 265 .

From (1) one can immediately compute that the probability that a random permutation has no fixed points is approximately $1 / e$. Another exercise that often accompanies counting derangements is to prove the following two term recurrence relation for $n \geqslant 2$,

$$
d_{n}=(n-1)\left(d_{n-1}+d_{n-2}\right),
$$

\footnotetext{
*Partially supported by NSF Mathematical Sciences Postdoctoral Research Fellowship DMS-0703567
} 
with initial conditions $d_{0}=1$ and $d_{1}=0$; see [20]. From (2) one can derive the following single term recurrence for derangement numbers,

$$
d_{n}=n d_{n-1}+(-1)^{n} .
$$

Recently, Gordon and McMahon [13] considered isometries of the $n$-dimensional hypercube that leave no facet unmoved. Algebraically, such an isometry is an element $\sigma$ of the hyperoctahedral group $B_{n}$ for which $\sigma(i) \neq i$ for any $i$. Combinatorially, the problem then is to enumerate $n \times n$ matrices with entries from $\{0, \pm 1\}$ such that each row and column has exactly one nonzero entry and no diagonal entry equals 1 . Gordon and McMahon derive a formula for the number of facet derangements similar to (1), an expression of facet derangements in terms of permutation derangements, and recurrence relations for facet derangements similar to (2) and (3).

In Section 2, we consider elements $\sigma$ in the wreath product $\mathcal{C}_{r} 2 \mathfrak{S}_{n}$, where $\mathcal{C}_{r}$ is the finite cyclic group of order $r$ and $\mathfrak{S}_{n}$ is the symmetric group on $n$ objects. A cyclic derangement is an element of $\mathcal{C}_{r}<\mathfrak{S}_{n}$ with no fixed point. Denote the set of cyclic derangements of $\mathcal{C}_{r}<\mathfrak{S}_{n}$ by $\mathfrak{D}_{n}^{(r)}$, and denote their number by $d_{n}^{(r)}=\left|\mathfrak{D}_{n}^{(r)}\right|$. Combinatorially, $d_{n}^{(r)}$ is also the number of matrices with entries from $\left\{0,1, \zeta, \ldots, \zeta^{r-1}\right\}$, where $\zeta$ is a primitive $r$ th root of unity, such that each row and each column has exactly one nonzero entry and no diagonal entry equals 1 . We derive a formula for $d_{n}^{(r)}$ that specializes to (1) when $r=1$ and to the Gordon-McMahon formula for facet derangements when $r=2$. We also give an expression for $d_{n}^{(r)}$ in terms of $d_{n}$ as well as a two recurrence relations specializing to (2) and (3) when $r=1$.

Gessel [12] introduced a $q$-analog for derangements of $\mathfrak{S}_{n}, q$-counted by the major index, that has applications to character theory [17]. In Section 3, we give a $q, t$-analog for cyclic derangements of $\mathcal{C}_{r} \prec \mathfrak{S}_{n} q$-counted by a generalization of major index and $t$ counted by signs that specializes to Gessel's formula at $r=1$ and $t=1$. Generalizing results in Section 2, we show that the cyclic $q$, $t$-derangements satisfy natural $q, t$-analogs of (1), (2) and (3). These results also generalize formulas of Garsia and Remmel [11] who first introduced $q$-analogs for (2) and (3) for $r=1$ using a different (though equidistributed) permutation statistic. The analogs we present are similar to results of Chow [5] and Foata and Han [9] when $r=2$, though the statistic we use differs slightly.

Brenti [1] gave another $q$-analog for derangements of $\mathfrak{S}_{n} q$-counted by weak excedances and conjectured many nice properties for these numbers that were later proved by Canfield (unpublished) and Zhang [22]. More recently, Chow [6] and Chen, Tang and Zhao [4] independently extended these results to derangements of the hyperoctahedral group. In Section 4 , we show that these results are special cases of cyclic derangements of $\mathcal{C}_{r} \succ \mathfrak{S}_{n}$ $q$-counted by a generalization of weak excedances.

The proofs for all of these generalizations are combinatorial, following the same reasoning as the classical proofs of (1) and (2). This suggests that studying derangements in this more general setting is very natural. In Section 5, we discuss possible directions for further study generalizing other results for permutations derangements. 


\section{Cyclic derangements}

Let $\mathfrak{S}_{n}$ denote the symmetric group of permutations of a set of $n$ objects, and let $\mathcal{C}_{r}$ denote the cyclic group of order $r$. The wreath product $\mathcal{C}_{r} \prec \mathfrak{S}_{n}$ is the semi-direct product $\left(\mathcal{C}_{r}\right)^{\times n} \rtimes \mathfrak{S}_{n}$, where $\mathfrak{S}_{n}$ acts on $n$ copies of $\mathcal{C}_{r}$ by permuting the coordinates. Let $\zeta$ be a generator for $\mathcal{C}_{r}$, e.g. take $\zeta$ to be a primitive $r$ th root of unity. We regard an element $\sigma \in \mathcal{C}_{r} \backslash \mathfrak{S}_{n}$ as a word $\sigma=\left(\epsilon_{1} s_{1}, \ldots, \epsilon_{n} s_{n}\right)$ where $\epsilon_{i} \in\left\{1, \zeta, \ldots, \zeta^{r-1}\right\}$ and $\left\{s_{1}, \ldots, s_{n}\right\}=\{1, \ldots, n\}$.

In this section we show that all of the usual formulas and proofs for classical derangement numbers generalize to these wreath products. We begin with (1), giving the following formula for the number of cyclic derangements. The two proofs below are essentially the same, though the first is slightly more direct while the latter will be useful for establishing $q$ and $q, t$ analogs.

Theorem 2.1. The number of cyclic derangements in $\mathcal{C}_{r} \prec \mathfrak{S}_{n}$ is given by

$$
d_{n}^{(r)}=r^{n} n ! \sum_{i=0}^{n} \frac{(-1)^{i}}{r^{i} i !} .
$$

Inclusion-Exclusion Proof. Let $A_{i}$ be the set of $\sigma \in \mathcal{C}_{r} \prec \mathfrak{S}_{n}$ such that $\sigma_{i}=+1 \cdot i$. Then $\left|A_{j_{1}} \cap \cdots \cap A_{j_{i}}\right|=r^{n-i}(n-i)$ !, since the positions $j_{1}, \ldots, j_{i}$ are determined and the remaining $n-i$ positions may be chosen arbitrarily. Therefore by the Inclusion-Exclusion formula, we have

$$
\begin{aligned}
\left|\mathfrak{D}_{n}^{(r)}\right| & =\mid \mathcal{C}_{r}\left\langle\mathfrak{S}_{n}|-| A_{1} \cup \cdots \cup A_{n}\right| \\
& =r^{n} n !-\sum_{i=1}^{n} \sum_{j_{1}<\cdots<j_{i}}(-1)^{i-1}\left|A_{j_{1}} \cap \cdots \cap A_{j_{i}}\right| \\
& =\sum_{i=0}^{n}\left(\begin{array}{c}
n \\
i
\end{array}\right)(-1)^{i} r^{n-i}(n-i) !=r^{n} n ! \sum_{i=0}^{n} \frac{(-1)^{i}}{r^{i} i !} .
\end{aligned}
$$

Möbius Inversion Proof. For $S=\left\{s_{1}<s_{2}<\cdots<s_{m}\right\} \subseteq[n]$ and $\sigma \in \mathcal{C}_{r}\left\langle\mathfrak{S}_{S}\right.$, define the reduction of $\sigma$ to be the permutation in $\mathcal{C}_{r}$ 々 $\mathfrak{S}_{m}$ that replaces $\epsilon_{i} s_{i}$ with $\epsilon_{i} i$. If $\sigma \in \mathcal{C}_{r}>\mathfrak{S}_{n}$ has exactly $k$ fixed points, then define $\operatorname{dp}(\sigma) \in \mathfrak{D}_{n-k}^{(r)}$ to be the reduction of $\sigma$ to the non-fixed points. For example, $\operatorname{dp}(5314762)=$ reduction of $53172=43152$ and any signs are carried over.

The map dp is easily seen to be an $\left(\begin{array}{l}n \\ k\end{array}\right)$ to 1 mapping of cyclic permutations with exactly $k$ fixed points onto $\mathfrak{D}_{n-k}^{(r)}$. Therefore

$$
r^{n} n !=\sum_{k=0}^{n}\left(\begin{array}{l}
n \\
k
\end{array}\right) d_{n-k}^{(r)} .
$$

The theorem now follows by Möbius inversion [20]. 
An immediate consequence of Theorem 2.1 is that the probability that a random element of $\mathcal{C}_{r} \geq \mathfrak{S}_{n}$ is a derangement is approximately $e^{-1 / r}$. This verifies the intuition that as $n$ and $r$ grow, most elements of $\mathcal{C}_{r}<\mathfrak{S}_{n}$ are in fact derangements. Table 1 gives values for $d_{n}^{(r)}$ for $r \leqslant 5$ and $n \leqslant 6$.

Table 1: Cyclic derangement numbers $d_{n}^{(r)}$ for $r \leqslant 5, n \leqslant 6$.

\begin{tabular}{c|rrrrrrr}
$r \backslash n$ & 0 & 1 & 2 & 3 & 4 & 5 & 6 \\
\hline 1 & 1 & 0 & 1 & 2 & 9 & 44 & 265 \\
2 & 1 & 1 & 5 & 29 & 233 & 2329 & 27949 \\
3 & 1 & 2 & 12 & 116 & 1393 & 20894 & 376093 \\
4 & 1 & 3 & 25 & 299 & 4785 & 95699 & 2296777 \\
5 & 1 & 4 & 41 & 614 & 12281 & 307024 & 9210721
\end{tabular}

We also have the following generalization of [13](Proposition 3.2), giving a formula relating the number of cyclic derangements with the number of permutation derangements.

Proposition 2.2. For $r \geqslant 2$ we have

$$
d_{n}^{(r)}=\sum_{i=0}^{n}\left(\begin{array}{c}
n \\
i
\end{array}\right) r^{i}(r-1)^{n-i} d_{i}
$$

where $d_{i}=\left|\mathfrak{D}_{i}\right|$ is the number of derangements in $\mathfrak{S}_{i}$.

Proof. For $S \subset\{1,2, \ldots, n\}$ of size $i$, the number of derangements $\sigma \in \mathcal{C}_{r}$ 々 $\mathfrak{S}_{n}$ with $|\sigma(j)|=j$ if and only if $j \notin S$ is equal to $d_{i} r^{i}$ (choose a permutation derangement of these indices and a sign for each) times $(r-1)^{n-i}$ (choose a nonzero sign for indices $k$ such that $\left.\left|\sigma_{k}\right|=k\right)$. There are $\left(\begin{array}{l}n \\ i\end{array}\right)$ choices for each such $S$, thereby proving (6).

Gordon and McMahon [13] observed that for $r=2$, the expression in (6) is precisely the rising 2-binomial transform of the permutation derangement numbers as defined by Spivey and Steil [18]. In general, this formula gives an interpretation for the mixed rising $r$-binomial transform and falling $(r-1)$-binomial transform of the permutation derangements numbers.

The following two term recurrence relation for cyclic derangements generalizes (2). We give two proofs of this recurrence, one generalizing the classical combinatorial proof of (2) and the other using the exponential generating function for cyclic derangements.

Theorem 2.3. For $n \geqslant 2$, the number of cyclic derangements satisfies

$$
d_{n}^{(r)}=(r n-1) d_{n-1}^{(r)}+r(n-1) d_{n-2}^{(r)},
$$

with initial conditions $d_{0}^{(r)}=1$ and $d_{1}^{(r)}=r-1$. 
Combinatorial Proof. For $\sigma \in \mathfrak{D}_{n}^{(r)}$, consider the cycle decomposition of underlying permutation $|\sigma| \in \mathfrak{S}_{n}$. There are three cases to consider. Firstly, if $n$ is in a cycle of length one, then there are $r-1$ choices for $\epsilon_{n} \neq 1$ and $d_{n-1}^{(r)}$ choices for a derangement of the remaining $n-1$ letters. If $n$ is in a cycle of length two in $|\sigma|$, then $\epsilon_{n}$ may be chosen freely in $r$ ways, there are $(n-1)$ choices for the other occupant of this two-cycle in $|\sigma|$ and $d_{n-2}^{(r)}$ choices for a cyclic derangement of the remaining $n-2$ letters. Finally, if $n$ is in a cycle of length three or more, then there are $r$ choices for $\epsilon_{n}, n-1$ possible positions for $n$ in $|\sigma|$, and $d_{n-1}^{(r)}$ choices for a derangement of the remaining $n-1$ letters. Combining these cases, we have

$$
d_{n}^{(r)}=(r-1) d_{n-1}^{(r)}+r(n-1) d_{n-2}^{(r)}+r(n-1) d_{n-1}^{(r)},
$$

from which (7) now follows.

Algebraic Proof. First note that for fixed $r$,

$$
\begin{aligned}
\frac{e^{-x}}{1-r x} & =\left(\sum_{i \geqslant 0} \frac{(-1)^{i}}{i !} x^{i}\right)\left(\sum_{j \geqslant 0} r^{j} x^{j}\right) \\
& =\sum_{n \geqslant 0} \sum_{i+j=n}\left(\begin{array}{c}
n \\
i
\end{array}\right) \frac{(-1)^{i} r^{j}}{i !} x^{n}=\sum_{n \geqslant 0} d_{n}^{(r)} \frac{x^{n}}{n !}
\end{aligned}
$$

is the exponential generating function for the number of cyclic derangements. Denoting this function by $D^{(r)}(x)$, we compute

$$
\begin{aligned}
& \sum\left((r n-1) d_{n-1}^{(r)}+(r n-r) d_{n-2}^{(r)}\right) \frac{x^{n}}{n !} \\
& =r \sum d_{n-1}^{(r)} \frac{x^{n}}{(n-1) !}-\sum d_{n-1}^{(r)} \frac{x^{n}}{n !}+r \sum d_{n-2}^{(r)} \frac{x^{n}}{(n-1) !}-r \sum d_{n-2}^{(r)} \frac{x^{n}}{n !} \\
& =r x D^{(r)}(x)-\int D^{(r)}(x)+r x \int D^{(r)}(x)-r \iint D^{(r)}(x)=D^{(r)}(x),
\end{aligned}
$$

from which the recurrence now follows.

Finally, we have the following simple recurrence relation generalizing (3).

Corollary 2.4. For $n \geqslant 1$, the number of cyclic derangements satisfies

$$
d_{n}^{(r)}=r n d_{n-1}^{(r)}+(-1)^{n},
$$

with initial condition $d_{0}^{(r)}=1$.

This recurrence follows by induction from the formula in Theorem 2.1 or the two term recurrence in Theorem 2.3, though it would be nice to have a direct combinatorial proof similar to that of Remmel [16] for the case $r=1$. 


\section{Cyclic $q, t$-derangements by major index}

Gessel [12] derived a $q$-analog for the number of permutation derangements as a corollary to a generating function formula for counting permutations in $\mathfrak{S}_{n}$ by descents, major index and cycle structure. In order to state Gessel's formula, we begin by recalling the $q$-analog of a positive integer $i$ given by $[i]_{q}=1+q+\cdots+q^{i-1}$. In the same vein, we also have $[i]_{q} !=[i]_{q}[i-1]_{q} \cdots[1]_{q}$, where $[0]_{q}$ ! is defined to be 1 .

For a permutation $\sigma \in \mathfrak{S}_{n}$, the descent set of $\sigma$, denoted by $\operatorname{Des}(\sigma)$, is given by $\operatorname{Des}(\sigma)=\{i \mid \sigma(i)>\sigma(i+1)\}$. MacMahon [14] used the descent set to define a fundamental permutation statistic, called the major index and denoted by maj $(\sigma)$, given by

$\operatorname{maj}(\sigma)=\sum_{i \in \operatorname{Des}(\sigma)} i$. Finally, recall MacMahon's formula [14] for $q$-counting permutations by major index,

$$
\sum_{\sigma \in \mathfrak{S}_{n}} q^{\operatorname{maj}(\sigma)}=[n]_{q} !
$$

Along these lines, define the $q$-derangement numbers, denoted by $d_{n}(q)$, by

$$
d_{n}(q)=\sum_{\sigma \in \mathfrak{D}_{n}} q^{\operatorname{maj}(\sigma)}
$$

Gessel showed that the $q$-derangement numbers for $\mathfrak{S}_{n}$ are given by

$$
d_{n}(q)=[n]_{q} ! \sum_{i=0}^{n} \frac{(-1)^{i}}{[i]_{q} !} q^{\left(\begin{array}{c}
i \\
2
\end{array}\right)} .
$$

A nice bijective proof of (10) is given by Wachs in [21], where she constructs a descentpreserving bijection between permutations with specified fixed points and shuffles of two permutations and then makes use of a formula of Garsia and Gessel [10] for $q$-counting shuffles. Garsia and Remmel [11] also studied $q$-derangement numbers using the inversion statistic which is known to be equi-distributed with major index.

Gessel's formula was generalized to the hyperoctahedral group by Chow [5] with further results by Foata and Han [9] using the flag major index statistic. Faliharimalala and Zeng [8] recently found a generalization of $(10)$ to $\mathcal{C}_{r}<\mathfrak{S}_{n}$ also using flag major index Here, we give a different generalization to $\mathcal{C}_{r}<\mathfrak{S}_{n}$ by $q$-counting with a different major index statistic and $t$-counting by signs.

We begin with a generalized notion of descents derived from the following total order on elements of $\left(\mathcal{C}_{r} \times[n]\right) \cup\{0\}$ :

$$
\zeta^{r-1} n<\cdots<\zeta n<\zeta^{r-1}(n-1)<\cdots<\zeta 1<0<1<2<\cdots<n
$$

For $\sigma \in \mathcal{C}_{r} \prec \mathfrak{S}_{n}$, an index $0 \leqslant i<n$ is a descent of $\sigma$ if $\sigma_{i}>\sigma_{i+1}$ with respect to this total ordering, where we set $\sigma_{0}=0$. Note that for $\sigma \in \mathfrak{S}_{n}$, this definition agrees with the classical one. As with permutations, define the major index of $\sigma$ by $\operatorname{maj}(\sigma)=\sum_{i \in \operatorname{Des}(\sigma)} i$. We also want to track the signs of the letters of $\sigma$, which we do with the statistic $\operatorname{sgn}(\sigma)$ defined by $\operatorname{sgn}(\sigma)=e_{1}+\cdots+e_{n}$, where $\sigma=\left(\zeta^{e_{1}} s_{1}, \ldots, \zeta^{e_{n}} s_{n}\right)$. This is a generalization of the same statistic introduced by Reiner in [15]. 
Remark 3.1. There is another total ordering on elements of $\left(\mathcal{C}_{r} \times[n]\right) \cup\{0\}$ that is equally as natural as the order given in (11), namely

$$
\zeta^{r-1} n<\cdots<\zeta^{r-1} 1<\zeta^{r-2} n<\cdots<\zeta 1<0<1<2<\cdots<n .
$$

While using this alternate order will result in a different descent set and major index for a given element of $\mathcal{C}_{r}<\mathfrak{S}_{n}$, the distribution of descent sets over $\mathcal{C}_{r} \prec \mathfrak{S}_{n}$ and even $\mathfrak{D}_{n}^{(r)}$ is the same with either ordering. In fact, there are many possible total orderings that refine the ordering on positive integers and yield the same distribution over $\mathcal{C}_{r} \prec \mathfrak{S}_{n}$ and $\mathfrak{D}_{n}^{(r)}$, since the proof of Theorem 3.2 carries through easily for these orderings as well. We have chosen to work with the ordering in (11) primarily to facilitate the combinatorial proof of Theorem 3.6.

A first test that these statistics are indeed natural is to see that the $q, t$ enumeration of elements of $\mathcal{C}_{r} \prec \mathfrak{S}_{n}$ by the major index and sign gives

$$
\sum_{\sigma \in \mathcal{C}_{r} \imath \mathfrak{S}_{n}} q^{\operatorname{maj}(\sigma)} t^{\operatorname{sgn}(\sigma)}=[r]_{t}^{n}[n]_{q} !
$$

which is a natural $(q, t)$-analog for $r^{n} n !=\left|\mathcal{C}_{r} \prec \mathfrak{S}_{n}\right|$.

Analogous to (9), define the cyclic $(q, t)$-derangement numbers by

$$
d_{n}^{(r)}(q, t)=\sum_{\sigma \in \mathfrak{D}_{n}^{(r)}} q^{\mathrm{maj}(\sigma)} t^{\operatorname{sgn}(\sigma)} .
$$

In particular, $d_{n}^{(1)}(q, t)=d_{n}(q)$ as defined in (9). In general, we have the following $(q, t)$ analog of (4) that specializes to (10) when $r=1$.

Theorem 3.2. The cyclic $(q, t)$-derangement numbers are given by

$$
d_{n}^{(r)}(q, t)=[r]_{t}^{n}[n]_{q} ! \sum_{i=0}^{n} \frac{(-1)^{i}}{[r]_{t}^{i}[i]_{q} !} q^{\left(\begin{array}{c}
i \\
2
\end{array}\right)}
$$

The proof of Theorem 3.2 is completely analogous to Wachs's proof [21] for $\mathfrak{S}_{n}$ which generalizes the second proof of Theorem 2.1. To begin, we define a map $\varphi$ that is a sort of inverse to the map dp. Say that $\sigma_{i}$ is a subcedant of $\sigma$ if $\sigma_{i}<i$ with respect to the total order in (11), and let $\operatorname{sub}(\sigma)$ denote the number of subcedants of $\sigma$. For $\sigma \in \mathcal{C}_{r}$ 々 $\mathfrak{S}_{m}$, let $s_{1}<\cdots<s_{\operatorname{sub}(\sigma)}$ be the absolute values of subcedants of $\sigma$. If $\sigma$ has $k$ fixed points, let $f_{1}<\cdots<f_{k}$ be the fixed points of $\sigma$. Finally, let $x_{1}>\cdots>x_{m-\operatorname{sub}(\sigma)-k}$ be the remaining letters in $[m]$. For fixed $n, \varphi(\sigma)$ is obtained from $\sigma$ by the following replacements:

$$
\epsilon_{i} s_{i} \mapsto \epsilon_{i} i \quad f_{i} \mapsto i+\operatorname{sub}(\sigma) \quad x_{i} \mapsto n-i+1 .
$$

For example, for $n=8$ we have $\varphi(3,2,-6,5,4,-1)=(7,4,-3,8,2,-1)$.

For disjoint sets $A$ and $B$, a shuffle of $\alpha \in \mathcal{C}_{r} \succ \mathfrak{S}_{A}$ and $\beta \in \mathcal{C}_{r} \succ \mathfrak{S}_{B}$ is an element of $\mathcal{C}_{r}<\mathfrak{S}_{A \cup B}$ containing $\alpha$ and $\beta$ as complementary subwords. Let $\operatorname{Sh}(\alpha, \beta)$ denote the set of shuffles of $\alpha$ and $\beta$. Then we have the following generalization of [21](Theorem 2). 
Lemma 3.3. Let $\alpha \in \mathfrak{D}_{n-k}^{(r)}$ and $\gamma=(\operatorname{sub}(\alpha)+1, \ldots, \operatorname{sub}(\alpha)+k)$. Then the map $\varphi$ gives a bijection $\left\{\sigma \in \mathcal{C}_{r} \prec \mathfrak{S}_{n} \mid \operatorname{dp}(\sigma)=\alpha\right\} \stackrel{\sim}{\longrightarrow} \operatorname{Sh}(\varphi(\alpha), \gamma)$ such that $\operatorname{Des}(\varphi(\sigma))=\operatorname{Des}(\sigma)$ and $\operatorname{sgn}(\varphi(\sigma))=\operatorname{sgn}(\sigma)$.

Proof. The preservation of sgn is obvious by construction. To see that the descent set is preserved, note that $\epsilon_{i} \neq 1$ only if $\sigma_{i}$ is a subcedant and the relative order of subcedants, fixed points and the remaining letters is preserved by the map. It remains only to show that $\varphi$ is an invertible map with image $\operatorname{Sh}(\varphi(\alpha), \gamma)$. For this, the proof of [21](Theorem 2) carries through verbatim thanks to the total ordering in (11).

The only remaining ingredient to prove Theorem 3.2 is the formula of Garsia and Gessel [10] for $q$-counting shuffles. Though their theorem was stated only for $\mathfrak{S}_{n}$, the result holds in this more general setting.

Lemma 3.4. Let $\alpha$ and $\beta$ be cyclic permutations of lengths $a$ and $b$, respectively, and let $\operatorname{Sh}(\alpha, \beta)$ denotes the set of shuffles of $\alpha$ and $\beta$. Then

$$
\sum_{\sigma \in \operatorname{Sh}(\alpha, \beta)} q^{\operatorname{maj}(\sigma)} t^{\operatorname{sgn}(\sigma)}=\left[\begin{array}{c}
a+b \\
a
\end{array}\right]_{q} q^{\operatorname{maj}(\alpha)+\operatorname{maj}(\beta)} t^{\operatorname{sgn}(\alpha)+\operatorname{sgn}(\beta)} .
$$

Proof of Theorem 3.2. For $\gamma$ as in Lemma 3.3, observe $\operatorname{maj}(\gamma)=0=\operatorname{sgn}(\gamma)$. Thus applying Lemma 3.3 followed by Lemma 3.4 allows us to compute

$$
\begin{aligned}
{[r]_{t}^{n}[n]_{q} ! } & =\sum_{\sigma \in \mathcal{C}_{r} \imath \mathfrak{S}_{n}} q^{\operatorname{maj}(\sigma)} t^{\operatorname{sgn}(\sigma)} \\
& =\sum_{k=0}^{n} \sum_{\alpha \in \mathfrak{D}_{n-k}^{(r)}} \sum_{\mathrm{dp}(\sigma)=\alpha} q^{\operatorname{maj}(\sigma)} t^{\operatorname{sgn}(\sigma)} \\
& =\sum_{k=0}^{n} \sum_{\alpha \in \mathfrak{D}_{n-k}^{(r)}} \sum_{\sigma \in \operatorname{Sh}(\varphi(\alpha), \gamma)} q^{\operatorname{maj}(\sigma)} t^{\operatorname{sgn}(\sigma)} \\
& =\sum_{k=0}^{n} \sum_{\alpha \in \mathfrak{D}_{n-k}^{(r)}}\left[\begin{array}{l}
n \\
k
\end{array}\right]_{q} q^{\operatorname{maj}(\alpha)} t^{\operatorname{sgn}(\alpha)} \\
& =\sum_{k=0}^{n}\left[\begin{array}{l}
n \\
k
\end{array}\right]_{q}^{(r)}(q, t) .
\end{aligned}
$$

Applying Möbius inversion to the resulting equation yields (15).

Proposition 2.2 also generalizes, though in order to prove the generalization we rely on the $(q, t)$-analog of the recurrence relation given in Theorem 3.6 below. It would be nice to have a combinatorial proof as well. 
Proposition 3.5. For $r \geqslant 2$ we have

$$
d_{n}^{(r)}(q, t)=\sum_{i=0}^{n}\left[\begin{array}{c}
n \\
i
\end{array}\right]_{q}[r]_{t}^{i}\left(\prod_{k=0}^{n-i-1}\left([r]_{t}-q^{k}\right)\right) d_{i}(q, t) .
$$

The recurrence relation (7) in Theorem 2.3 also has a natural $(q, t)$-analog. Note that this specializes to the formula of Garsia and Remmel [11] in the case $r=1$. The proof is combinatorial, though it would be nice to have a generating function proof as well.

Theorem 3.6. The cyclic $(q, t)$-derangement numbers satisfy

$$
d_{n}^{(r)}(q, t)=\left([r]_{t}[n]_{q}-q^{n-1}\right) d_{n-1}^{(r)}(q, t)+q^{n-1}[r]_{t}[n-1]_{q} d_{n-2}^{(r)}(q, t),
$$

with initial conditions $d_{0}^{(r)}(q, t)=1$ and $d_{1}^{(r)}(q, t)=[r]_{t}-1$.

Proof. As in the combinatorial proof of Theorem 2.3, consider the cycle decomposition of underlying permutation $|\sigma| \in \mathfrak{S}_{n}$. We consider the same three cases, this time tracking the major index and sign. If $n$ is in a cycle of length one, then the $r-1$ choices for $\epsilon_{n} \neq 1$ contribute $t[r-1]_{t}$, and there will necessarily be a descent in position $n-1$, thus contributing $q^{n-1}$. This case then contributes

$$
t[r-1]_{t} q^{n-1} d_{n-1}^{(r)}(q, t) .
$$

If $n$ is in a cycle of length two in $|\sigma|$, then $\epsilon_{n}$ is arbitrary contributing $[r]_{t}$, and the $n-1$ choices for the other occupant of the cycle will add at least $n-1$ to the major index beyond the major index of the permutation with these two letters removed. This contributes a term of $q^{n-1}[n-1]_{q}$, making the total contribution

$$
[r]_{t} q^{n-1}[n-1]_{q} d_{n-2}^{(r)}(q, t) .
$$

Finally, if $n$ is in a cycle of length three or more, then each of the $n-1$ possible positions for $n$ in $|\sigma|$ increases the major index by one, contributing a factor of $[n-1]_{q}$. The $r$ choices for $\epsilon_{n}$ again contribute $[r]_{t}$, giving a total of

$$
[r]_{t}[n-1]_{q} d_{n-1}^{(r)}(q, t) .
$$

Adding these three cases yields (18).

As before, we may use induction and (18) to derive the following single term recurrence relation for cyclic $(q, t)$-derangements generalizing (8) of Corollary 2.4.

Corollary 3.7. The cyclic $(q, t)$-derangement numbers satisfy

$$
d_{n}^{(r)}(q, t)=[r]_{t}[n]_{q} d_{n-1}^{(r)}(q, t)+(-1)^{n} q^{\left(\begin{array}{c}
n \\
2
\end{array}\right)},
$$

with initial condition $d_{0}^{(r)}(q, t)=1$. 


\section{Cyclic $q$-derangements by weak excedances}

Brenti [1] studied a different $q$-analog of derangement numbers, defined by $q$-counting derangements by the number of weak excedances, in order to study certain symmetric functions introduced by Stanley [19]. Later, Brenti [3] defined weak excedances for the signed permutations to study analogous functions for the hyperoctahedral group. Further results were discovered by Zhang [22] and Chow [6] and Chen, Tang and Zhao [4] for the symmetric and hyperoctahedral groups. Below we extend these results to the wreath product $\mathcal{C}_{r} \prec \mathfrak{S}_{n}$.

Recall that an index $i$ is a weak excedant of $\sigma$ if $\sigma(i)=i$ or $\sigma^{2}(i)>\sigma(i)$. It has long been known that the number of descents and the number of weak excedances are equi-distributed over $\mathfrak{S}_{n}$ and that both give the Eulerian polynomials $A_{n}(q)$ :

$$
A_{n}(q) \stackrel{\text { def }}{=} \sum_{\sigma \in \mathfrak{S}_{n}} q^{\operatorname{des}(\sigma)+1}=\sum_{\sigma \in \mathfrak{S}_{n}} q^{\operatorname{exc}(\sigma)},
$$

where $\operatorname{des}(\sigma)$ is the number of descents of $\sigma$ and $\operatorname{exc}(\sigma)$ is the number of weak excedances of $\sigma$. We generalize these statistics to $\mathcal{C}_{r}<\mathfrak{S}_{n}$ by saying $1 \leqslant i \leqslant n$ is a weak excedant of $\sigma \in \mathcal{C}_{r} \succ \mathfrak{S}_{n}$ if $\sigma(i)=i$ or if $|\sigma(i)| \neq i$ and $\sigma^{2}(i)>\sigma(i)$ with respect to the total order in (11).

As with the number of descents, this statistic agrees with the classical number of weak excedances for permutations and Brenti's statistic for signed permutations. Moreover, the equi-distribution of the number of non-descents and the number of weak excedances holds in $\mathcal{C}_{r}<\mathfrak{S}_{n}$, and the same bijective proof using canonical cycle form [20] holds in this setting. Therefore define the cyclic Eulerian polynomial $A_{n}^{(r)}(q)$ by

$$
A_{n}^{(r)}(q)=\sum_{\sigma \in \mathcal{C}_{r} \imath \mathfrak{S}_{n}} q^{n-\operatorname{des}(\sigma)}=\sum_{\sigma \in \mathcal{C}_{r} \imath \mathfrak{S}_{n}} q^{\operatorname{exc}(\sigma)}
$$

Note that $A_{n}^{(r)}(q)$ is palindromic for $r \leqslant 2$, i.e. $A_{n}^{(r)}(q)=q^{n} A_{n}^{(r)}(1 / q)$. In particular, (21) specializes to (20) when $r=1$ and to Brenti's type B Eulerian polynomial when $r=2$. For $r \geqslant 3$, the cyclic Eulerian polynomial is not palindromic.

Restricting to the set of cyclic derangements of $\mathcal{C}_{r}<\mathfrak{S}_{n}$, the number of descents and weak excedances are no longer equi-distributed, even for $r=1$. Define the cyclic $q$-derangement polynomials $D_{n}^{(r)}(q)$ by

$$
D_{n}^{(r)}(q)=\sum_{\sigma \in \mathfrak{D}_{n}^{(r)}} q^{\operatorname{exc}(\sigma)} .
$$

We justify this definition with the following two-term recurrence relation generalizing Theorem 2.3. Note that this reduces to the result of Brenti [1] when $r=1$ and the analog for the hyperoctahedral group $[6,4]$ when $r=2$.

Theorem 4.1. For $n \geqslant 2$, the cyclic $q$-derangement polynomials satisfy

$$
D_{n}^{(r)}=(n-1) r q\left(D_{n-1}^{(r)}+D_{n-2}^{(r)}\right)+(r-1) D_{n-1}^{(r)}+r q(1-q) \frac{\mathrm{d}}{\mathrm{d} q} D_{n-1}^{(r)}
$$


with initial conditions $D_{0}^{(r)}(q)=1$ and $D_{1}^{(r)}(q)=r-1$.

Proof. As in the combinatorial proof of Theorem 2.3, consider the cycle decomposition of underlying permutation $|\sigma| \in \mathfrak{S}_{n}$. We consider the same three cases, now tracking the number of weak excedances. If $n$ is in a cycle of length one, then there are $r-1$ choices for $\epsilon_{n} \neq 1$, and $n$ is not a weak excedant of $\sigma$. Removing this cycle leaves a cyclic derangement in $\mathfrak{D}_{n-1}^{(r)}$ with the same number of weak excedances, thus contributing

$$
(r-1) D_{n-1}^{(r)}(q) .
$$

If $n$ is in a cycle of length two in $|\sigma|$, then $\epsilon_{n}$ is arbitrary, and there are $n-1$ choices for the other occupant of the cycle, say $k$. Moreover, exactly one of $k$ and $n$ will be a weak excedant, and so the contribution in this case is

$$
r(n-1) q D_{n-2}^{(r)}(q) .
$$

Finally, if $n$ is in a cycle of length three or more, then $\epsilon_{n}$ is again arbitrary, but the affect on weak excedances for the $n-1$ possible placements of $n$ is more subtle. If $n$ is placed between $i$ and $j$ with $\sigma(j)=\sigma(\sigma(i))>\sigma(i)$, then the number of (weak) excedances remains unchanged when inserting $n$. However, if $n$ is placed between $i$ and $j$ with $\sigma(j)=\sigma(\sigma(i))<\sigma(i)$, then the insertion of $n$ creates a new (weak) excedant. Therefore we have

$$
\begin{array}{r}
r \sum_{\tau \in \mathfrak{D}_{n-1}^{(r)}}\left(\operatorname{exc}(\tau) q^{\operatorname{exc}(\tau)}+(n-1-\operatorname{exc}(\tau)) q^{\operatorname{exc}(\tau)+1}\right) \\
\quad=r(n-1) q D_{n-1}^{(r)}(q)+r(1-q) q \frac{\mathrm{d}}{\mathrm{d} q} D_{n-1}^{(r)}(q) .
\end{array}
$$

Adding these three cases yields (23).

Using (23), we can also compute the exponential generating function of the cyclic Eulerian polynomials and cyclic $q$-derangement polynomials.

Proposition 4.2. For $r \geqslant 1$, we have

$$
\sum_{n \geqslant 0} A_{n}^{(r)}(q) \frac{x^{n}}{n !}=\frac{(1-q) e^{x(1-q)}}{1-q e^{r x(1-q)}}
$$

and

$$
\sum_{n \geqslant 0} D_{n}^{(r)}(q) \frac{x^{n}}{n !}=\frac{(1-q) e^{x(r-1)}}{e^{q x x}-q e^{r x}} .
$$

Proof. Reversing the generating function proof of Theorem 2.3, it is straightforward to show that (25) satisfies the recurrence relation in (23). Enumerating elements of $\mathcal{C}_{r}$ ? $\mathfrak{S}_{n}$ by the number of fixed points yields

$$
A_{n}^{(r)}(q)=\sum_{k=0}^{n}\left(\begin{array}{l}
n \\
k
\end{array}\right) q^{k} D_{n-k}^{(r)}(q),
$$

from which (24) follows. 
Recall that a sequence $a_{0}, a_{1}, \ldots, a_{m}$ of real numbers is unimodal if for some $j$ we have $a_{0} \leqslant a_{1} \leqslant \cdots \leqslant a_{j} \geqslant a_{j+1} \geqslant \cdots \geqslant a_{m}$. A sequence is log-concave if $a_{i}^{2} \geqslant a_{i-1} a_{i+1}$ for all $i$. It is not difficult to show that a log-concave sequence of positive numbers is unimodal. More generally, a sequence is a Pólya frequency sequence if every minor of the (infinite) matrix $\left(a_{j-i}\right)$ is nonnegative, where we take $a_{k}=0$ for $k<0$ and $k>m$. Pólya frequency sequences arise often in combinatorics, and one of the fundamental results concerning them is the following.

Theorem 4.3. The roots of a polynomial $a_{0}+a_{1} x+\cdots+a_{m} x^{m}$ are all real and non-positive if and only if the sequence $a_{0}, a_{1}, \ldots, a_{m}$ is a Pólya frequency sequence.

Using (23) and Theorem 4.3, we will show that for fixed $n$ and $r$, the sequence $a_{m}=$ $\#\left\{\sigma \in \mathfrak{D}_{n}^{(r)} \mid \operatorname{exc}(\sigma)=m\right\}$ is unimodal and log-concave.

Theorem 4.4. For $n \geqslant 2$, the roots of the cyclic q-derangement polynomial $D_{n}^{(r)}(q)$ interlace the roots of $D_{n+1}^{(r)}(q)$. In particular, they are distinct, non-positive real numbers and the coefficients of $D_{n}^{(r)}(q)$ are a Pólya frequency sequence.

Proof. We proceed by induction on $n$. From (23), we have that the leading term of $D_{n}^{(r)}(q)$ is $r^{n} q^{n-1}$ and the constant term is $(r-1)^{n}$. In particular,

$$
D_{2}^{(r)}(q)=r^{2} q+(r-1)^{2} \quad \text { and } \quad D_{3}^{(r)}(q)=r^{3} q^{2}+(4 r-3) r^{2} q+(r-1)^{3} .
$$

Thus the root of $D_{2}^{(r)}(q)$ is $-(r-1)^{2} / r^{2}$, which is indeed real and non-positive and lies between the two distinct negative real roots of $D_{3}^{(r)}(q)$. This demonstrates the base case, so assume the result for $n-1 \geqslant 2$.

Let $0>q_{1}>q_{2}>\cdots>q_{n-2}$ be the simple roots of $D_{n-1}^{(r)}(q)$. It is straightforward to show that $\left(D_{n-1}^{(r)}\right)^{\prime}\left(q_{i}\right)$ has sign $(-1)^{i+1}$, and by induction, the sign of $D_{n-2}^{(r)}\left(q_{i}\right)$ is also $(-1)^{i+1}$ as the roots are interlaced. By (23), we have

$$
D_{n}^{(r)}\left(q_{i}\right)=(n-1) r q_{i} D_{n-2}^{(r)}\left(q_{i}\right)+r q_{i}\left(1-q_{i}\right)\left(D_{n-1}^{(r)}\right)^{\prime}\left(q_{i}\right)
$$

from which it follows that the sign of $D_{n}^{(r)}\left(q_{i}\right)$ is $(-1)^{i}$. Given the leading and constant terms of $D_{n}^{(r)}(q)$, by the Intermediate Value Theorem, the roots of $D_{n}^{(r)}(q)$ are interlaced with $0, q_{1}, \ldots, q_{n-2},-\infty$.

\section{$5 \quad$ Further directions}

Binomial transforms. Spivey and Steil [18] define two variants of the binomial transform of a sequence: the rising $k$-binomial transform and the falling $k$-binomial transform given by

$$
r_{n}=\sum_{i=0}^{n}\left(\begin{array}{l}
n \\
i
\end{array}\right) k^{i} a_{i} \text { and } f_{n}=\sum_{i=0}^{n}\left(\begin{array}{l}
n \\
i
\end{array}\right) k^{n-i} a_{i}
$$


respectively. Gordon and McMahon noted that the number of derangements in the hyperoctahedral group gives the rising 2-binomial transform of the derangement numbers for $\mathfrak{S}_{n}$. More generally, Proposition 2.2 shows that the cyclic derangement numbers $d_{n}^{(r)}$ give a mixed version of the rising $r$-binomial transform and falling $(r-1)$-binomial transform of $d_{n}$. This new hybrid $k$-binomial transform may share many of the nice properties of Spivey and Steil's transforms, including Hankel invariance and/or a simple description of the change in the exponential generating function. Further, it could be interesting to evaluate the expression in (6) for negative or even non-integer values of $k$. For instance, taking $k=1 / 2$ gives the binomial mean transform which is of some interest.

Limiting distributions. The explicit expression in (4) immediately gives approximations and asymptotics for the probability that a random element of $\mathcal{C}_{r}<\mathfrak{S}_{n}$ is a derangement. Moreover, this formula can be used to calculate the number of elements with a given number of fixed points, and so, too, can be used to calculate the expected number of fixed points of a random element. For instance, in $\mathfrak{S}_{n}$ it is know that the number of fixed points of a random permutation has a limiting Poisson distribution, and recent work by Diaconis, Fulman and Guralnick [7] has extended this to primitive actions of $\mathfrak{S}_{n}$. A natural extension would be use the combinatorics presented here to consider the imprimitive action of $\mathfrak{S}_{n}$ in $\mathcal{C}_{r}>\mathfrak{S}_{n}$.

Orthogonal idempotents. Another direction would be to generalize the work of Schocker [17] where he uses derangement numbers to construct $n$ mutually orthogonal idempotents in Solomon's descent algebra for $\mathfrak{S}_{n}$. In doing so, he also discovers a new proof of Gessel's formula for $q$-derangements. Thus extending these techniques to analogs of the descent algebra for $\mathcal{C}_{r} \prec \mathfrak{S}_{n}$ could also lead to new proofs of the formulae in Section 3.

Symmetric unimodal polynomials. In [2], Brenti used symmetric functions to define several new classes of symmetric unimodal polynomials. Brenti showed there is an explicit connection between these polynomials and the $q$-Eulerian polynomials and $q$-derangement polynomials of $\mathfrak{S}_{n}$ counted by weak excedances. Brenti generalized much of this work to the hyperoctahedral group, with additional results extended by Chow [6]. A natural question is to see if there exist analogs of these results for $\mathcal{C}_{r} \prec \mathfrak{S}_{n}$ involving the polynomials $A_{n}^{(r)}(q)$ and $D_{n}^{(r)}(q)$ studied in Section 4 .

\section{References}

[1] F. Brenti. Unimodal, log-concave and Pólya frequency sequences in combinatorics. Mem. Amer. Math. Soc., 81(413):viii+106, 1989.

[2] F. Brenti. Unimodal polynomials arising from symmetric functions. Proc. Amer. Math. Soc., 108(4):1133-1141, 1990.

[3] F. Brenti. q-Eulerian polynomials arising from Coxeter groups. European J. Combin., 15(5):417-441, 1994.

[4] W. Y. C. Chen, R. L. Tang, and A. F. Y. Zhao. Derangement polynomials and excedances of type B. Electron. J. Combin., 16(2):R15, 2009. 
[5] C.-O. Chow. On derangement polynomials of type B. Sém. Lothar. Combin., 55:Art. B55b, 6 pp. (electronic), 2006.

[6] C.-O. Chow. On derangement polynomials of type B. II. J. Combin. Theory Ser. A, 116(4):816-830, 2009.

[7] P. Diaconis, J. Fulman, and R. Guralnick. On fixed points of permutations. J. Algebraic Combin., 28(1):189-218, 2008.

[8] H. Faliharimalala and J. Zeng. Fix-Euler-Mahonian statistics on wreath products. Preprint, 2009.

[9] D. Foata and G.-N. Han. Signed words and permutations. IV. Fixed and pixed points. Israel J. Math., 163:217-240, 2008.

[10] A. M. Garsia and I. Gessel. Permutation statistics and partitions. Adv. in Math., 31(3):288-305, 1979.

[11] A. M. Garsia and J. Remmel. A combinatorial interpretation of $q$-derangement and q-Laguerre numbers. European J. Combin., 1(1):47-59, 1980.

[12] I. M. Gessel and C. Reutenauer. Counting permutations with given cycle structure and descent set. J. Combin. Theory Ser. A, 64(2):189-215, 1993.

[13] G. Gordon and E. McMahon. Moving faces to other places: Facet derangements. Amer. Math. Monthly. To appear.

[14] P. A. MacMahon. The Indices of Permutations and the Derivation Therefrom of Functions of a Single Variable Associated with the Permutations of any Assemblage of Objects. Amer. J. Math., 35(3):281-322, 1913.

[15] V. Reiner. Signed permutation statistics. European J. Combin., 14(6):553-567, 1993.

[16] J. B. Remmel. A note on a recursion for the number of derangements. European J. Combin., 4(4):371-374, 1983.

[17] M. Schocker. Idempotents for derangement numbers. Discrete Math., 269(1-3):239248, 2003.

[18] M. Z. Spivey and L. L. Steil. The $k$-binomial transforms and the Hankel transform. J. Integer Seq., 9(1):Article 06.1.1, 19 pp. (electronic), 2006.

[19] R. P. Stanley. Log-concave and unimodal sequences in algebra, combinatorics, and geometry. In Graph theory and its applications: East and West (Jinan, 1986), volume 576 of Ann. New York Acad. Sci., pages 500-535. New York Acad. Sci., New York, 1989.

[20] R. P. Stanley. Enumerative combinatorics. Vol. 1, volume 49 of Cambridge Studies in Advanced Mathematics. Cambridge University Press, Cambridge, 1997. With a foreword by Gian-Carlo Rota, Corrected reprint of the 1986 original.

[21] M. L. Wachs. On q-derangement numbers. Proc. Amer. Math. Soc., 106(1):273-278, 1989.

[22] X. Zhang. On q-derangement polynomials. In Combinatorics and graph theory '95, Vol. 1 (Hefei), pages 462-465. World Sci. Publ., River Edge, NJ, 1995. 